\title{
Hardware Development for Pervasive Healthcare Systems: Current Status and Future Directions
}

\author{
Amit Acharyya*, Koushik Maharatna ${ }^{\dagger}$ and Bashir M. Al-Hashimi ${ }^{\ddagger}$ \\ School of Electronics and Computer Science, \\ University of Southampton, Southampton - SO17 1BJ, United Kingdom \\ Email: $\left\{{ }^{*} \mathrm{aa} 07 \mathrm{r},{ }^{\dagger} \mathrm{km} 3,{ }^{\dagger} \mathrm{bmah}\right\} @$ ecs.soton.ac.uk
}

\begin{abstract}
This paper surveys ongoing research for pervasive healthcare system development, highlights the associated technical challenges and outlines some possible future topics that require innovative research. We present a conceptual high level system overview for pervasive healthcare including a resource constrained architecture for extracting fetal electrocardiogram from maternal composite abdominal signal as a case study.
\end{abstract}

\section{INTRODUCTION}

In recent years, rapid development in embedded technology, communication technology and sensor networks turn pervasive computing theoretical research into practical possibility [1]. It opened up significant opportunity in health monitoring system development and will help making the concept of personalized healthcare a reality [2]. Such system has tremendous potential to provide in-time care and safety interventions, lowering healthcare cost and allowing early detection of health related problems [3]. Though pervasive computing has attracted considerable academic research and industrial interest, still more research has to be done in pervasive healthcare [4].

In this paper, we present an overview of the current research trend for pervasive healthcare system, discuss associated technical challenges and outline some possible future research opportunities. We present a conceptual high-level architecture where low complexity specialized circuitry could be embedded within the sensor nodes for such a system. Some preliminary results of such specialized hardware design from our research are also shown here. The remainder of this paper is organized as follows. Section II gives an overview of the ongoing research in this domain. Section III discusses the proposed conceptual architecture and our main research focus. Section IV justifies the necessity of our proposed architecture with a case study on low-power architecture development for pervasive healthcare. Future research challenges are discussed in Section V.

\section{CURRENT RESEARCH Focus}

There is a large body of literatures existing in the field of pervasive computing. Depending upon current research thrust, recent works can be classified in three categories - system architecture, sensing and communication, and service.

\section{A. System Architecture}

Pervasive healthcare applications would require sensing, communication and services. This represents conflicting requirements and therefore numerous architectures have been proposed. Recently a generic reference architecture of pervasive computing is discussed in [1] on the basis of current status of it, and in conjunction with ideological and security mechanism. High level view of personalized pervasive healthcare is reported in [4]. An adaptable pervasive computing architecture is discussed in [5] where main research challenges have been identified as - pre-processing of the collected physiological signal to extract particular feature and analyzing the extracted data to make decision. A wide area mobile patient monitoring system architecture is discussed in [6]. According to this, the potential benefits of such system architecture are - continuous monitoring for chronically ill patients, better quality care and feedback for patients, increased medical capacity and reduced medical cost for patient care.

\section{B. Sensing and Communication}

In [7], 4C's - Computing, Communication, Cognition and Collaboration are identified as the basic requirement of technology development for pervasive computing. In recent years considerable amount of research has been performed on Computing and Communication - the first two of 4Cs assuming that the sensor nodes can only capture data and transmit to the central node which has adequate resources for computation. Accordingly emphasis is given towards developing new generation distributed wireless intelligent sensor system [8] which is convenient to use, noninvasive in nature and unobtrusive so that it does not affect the normal activity. One such effort is given in [9] where a real-time remote arrythmia monitoring system prototype has been developed in NASA. It describes the system architecture and implementation issues for such system from the perspective of wireless communication technology. [10] describes the management framework for comprehensive wireless patient monitoring. The main research focus is in reliable network architecture development. The applications and challenges of pervasive healthcare and wireless health monitoring are discussed in detail in [11] by the same author where important issues in communication layer including throughput, quality of service, network reliability, network coverage and traffic management are discussed. Recently an interesting research has been carried out in [12] to provide a common taxonomy of pervasive healthcare systems. The main aim of pervasive computing- to provide people with a more natural way to interact with technology and services by embedding computation into the environment as unobtrusively 
as possible [13]. [6] depicts the importance of medical sensors for remote health monitoring of the patient. In [14] and [15], taxonomies of Wireless Sensor Networks (WSN) models have been presented depending upon the different communication functions and classification is done based on data delivery models, network dynamics and communication protocol perspective. Survey on wireless sensor network has been done in [16]. [17] presents wireless integrated network sensors combining microsensor technology, low-power signal processing, computation and low-cost wireless networking in a compact system. The importance of signal processing in the wireless sensor networks has been described in [18] to reduce the number of bits transmitted over the communication channel. It has been mentioned that different optimum algorithms to solve different research problems related to signal processing are not efficient when implemented because the total power consumption exceeds their benefits. Power consumption has been identified as one of the most important constraint for designing sensor rich pervasive networks in [19] and [20]. [21] identifies the advantages of such pre-processing unit inside the sensor nodes as it makes the transmitter/ receiver coding/ decoding strategies optimum and reduces number of bits transmitted over communication channel. This reduces the burden on communication layer as well as consumes less power. [20] and [22] show that sensor nodes have limited energy resources and mainly depend on on-board energy (battery back-up) or harvested energy.

\section{Service}

[4] indicates that next generation of pervasive healthcare services must provide personalized solution across distributed networks, where care professionals or informal care givers can monitor an individual's wellbeing in their own home. This will accomplish the goal of independent living. Subject to user agreement, information can be sent to a variety of care providers. Security and authentication have been identified as main concern in service layer [4]. One important issue in this layer is - how to provide better healthcare services to an increasing number of people using limited financial and human resources [10]. The current and emerging pervasive computing technology could improve the overall quality of service for patients in both cities and rural areas, reduce the stress and strain on healthcare providers, while enhancing their productivity, retention and quality of life, reduce long term cost of healthcare services [10]. There should be communication link among hospital, ambulance and healthcare providers for getting emergency service. Healthcare professionals have to be trained [10]. Along with this longitudinal and clinical studies are needed to realize the benefits of pervasive healthcare [3].

It is clear from the above discussion that due to recent advances in Micro-Electro-Mechanical Systems (MEMS) and wireless communication, main research focus in wireless sensor network is concentrated mostly on sensing unit and transceiver designing. Power, area and memory - constraints in WSN limit the development of signal processing algorithms which are computationally intensive. But, continuous process- ing at the sensor node would drastically reduce the financial, computational, and management burden on communication system components, networks, and human resources. For these reasons, current research tends towards power-aware protocols and algorithm development. The open research issue is to design low-power, low-cost, tiny processing unit inside the sensor node which takes sensed signal, pre-processes it and collaborate with the transceiver [19].

\section{Proposed Conceptual Architecture}

Based on various architectures proposed in [1], [4], [5], [6], we believe, an effective system for pervasive healthcare to contain three layers - Sensor, Communication and Service. In the first layer a set of sensors (wearble or environmental) are deployed for collecting the vital signs of the patient under monitoring. These collected data are transmitted via communication layer to the central facility. WLAN/ cellular/ GSM/ 3G network could be used for serving this purpose. In service layer appropriate healthcare service can be informed to take action. Although this architecture may be effective, it faces the problem of transmitting significant amount of data over the communication layer and effective management of the data at the central facility. The quality of transmitted medical data cannot be compromised by any means, therefore research effort has been dedicated to improve bit error rate, reliability, network performance, coverage, quality and bandwidth in the communication layer [1], [5], [6]. Additionally maintenance of the data in the central facility needs intelligent database development. One possible solution, which our research is pursuing, is to embed intelligent signal processing circuitry in Sensor Layer itself which can monitor the patient's vital signs on a continuous basis. However this approach calls for simplified area and power constrained signal processing algorithm and architecture development for processing physiological data which is discussed next.

Among these three layers, our reseach is focussing on Layer 1 - Sensor Layer. We envisage a system where the specialized signal processing unit and corresponding intelligent decision making circuitry will be embedded inside the sensor layer within the sensor nodes for continuous monitoring of patient's vital signs. It can communicate with transceiver inside the sensor node to store processed data in home computer and only transmit the signal to the central facility through this transceiver when it finds departure of the vital sign's pattern from the normal pattern. Our research interest is to develop low-power, area and memory-efficient, and intelligent signal processing architecture for pervasive healthcare applications.

This design unit inside the Sensor Layer within the sensor node will check the trend of the vital sign from the received sensor data. If any abnormality is sensed, then only the central node will be communicated. Otherwise the processed medical data will be stored in the local computer using home wireless infrastructure. This approach will reduce the burden on the communication layer significantly. If required, the central facility can call for the data stored in the home computer and other relevant information over the communication layer for 


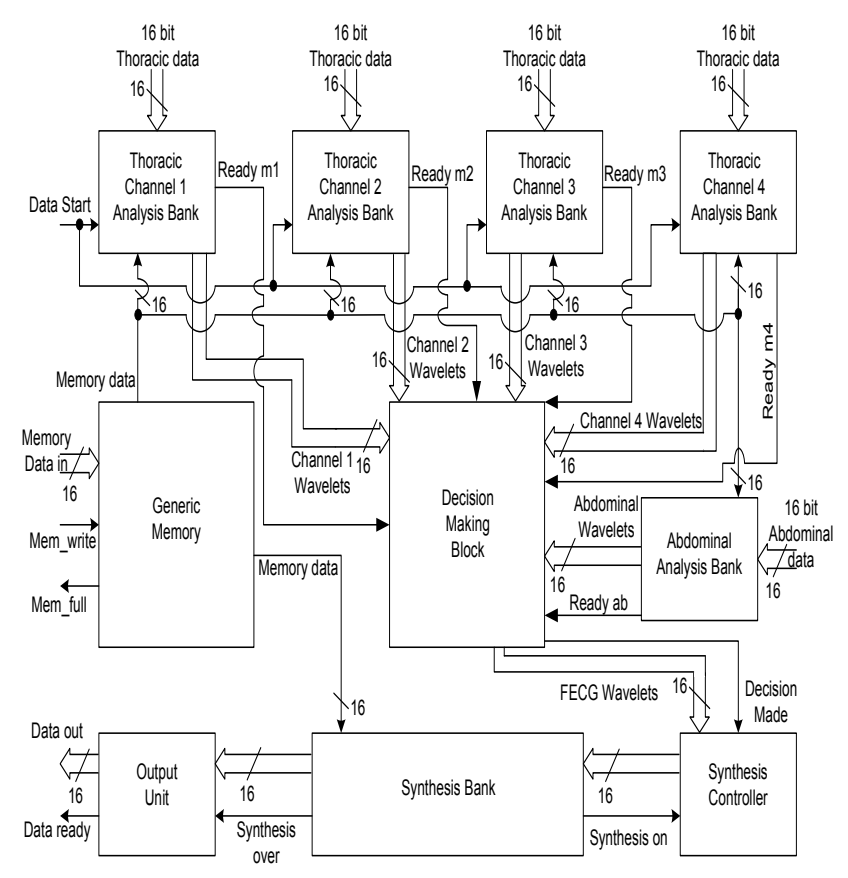

Fig. 1. Proposed VLSI architecture for FECG extraction

detail diagnosis of the vital signs. However, processing the physiological signals on a continuous basis in this type of infrastructure is an extremely challenging problem owing to the limited resources as mentioned in [18], [20] and [22]. To materialise such system, it is necessary to reduce the complexity of the required signal processing tasks ("light-weight signal processing") and associated architectural optimisation in such a way that each of the processing circuit consumes as small power as possible.

\section{CAse Study}

Here we consider one challenging task of signal processing in healthcare - extraction of Fetal Electrocardiogram (FECG) from abdominal composite signal of mother and will discuss its corresponding architecture from the perspective of pervasive healthcare.

Typically, the recorded signal, used for this purpose, is the mixture of weak FECG, a strong Maternal Electrocardiogram (MECG), and random contaminations due to other noncardiac sources [23]. A robust method of FECG extraction from abdominal composite signal has been proposed in [24]. This method deals with complex signal processing tasks including wavelet transform, fuzzification, reconstruction which increase the complexity of the circuit in terms of extensive memory and power requirement. Thus, we believe one to one mapping of this algorithm [24] into architecture may not fulfill resource constraints for pervasive healthcare. So, we focus on efficient algorithm to architecture mapping for this method to satisfy the constraints of pervasive healthcare. From the perspective of low power architecture development, reduction of number of multiplication and memory size are of paramount importance.
Fig. 1 presents the architectural overview of FECG extraction system. Multiplierless architecture of wavelet analysis and synthesis filter banks has been implemented using Distributed Arithmetic (DA) approach. But, in conventional DA based approach [25], power is dissipated by memory, shifters, adders/ subtractors and accumulator units and considerable amount of this dissipation occurs in memory. The shifters can be implemented by simple wire connections. So, main power consuming units are adders/ subtractors, memory and comparator logic unit. We have used this DA based approach in a modified way. This leads to significant reduction in memory requirement compared to the conventional DA based method [25]. The novelty of our design is the development of a memory-efficient, re-configurable, multiplierless, parameterized Discrete Wavelet Transform (DWT) and Inverese DWT (IDWT) architecture and corresponding decision-making circuitry which eliminates complex fuzzification as required in [24].

The architecture is designed assuming each incoming frame consists of 16 ECG samples each having 16 bits word-length because normally the number of bits per ECG sample varies from 12 to 36 [11]. The filter length is assumed to be 16 and each coefficient consists of 16 bits. As number of lead used of FECG extraction may vary [24], we have considered 5-channel input for our architecture where four channels get thoracic ECG and one is used to receive composite data. Fig. 1 shows the main design units - Analysis Bank, Decision Making Block, Generic Memory and Synthesis Bank along with Controller and Output unit. The Analysis Bank and Synthesis Bank correspond to DWT and IDWT respectively. While designing these blocks, although convolution-based method is exploited, we have implemented them using only adders/ subtractors, simple comparator logic and a generic memory unit and thereby making them multiplierless. This memory unit is designed by considering pre-computed combination of filter coefficients to save significant amount of memory. This model is generic so that it can be configured with any kind of wavelets. The concept of complex fuzzification is substituted by using simple decision-making logic. This block is implemented by assigning simple "token" attribute to particular wavelet and using comparison logic.

The generic memory is the most significant block in our architecture. It has been designed to serve two purposes first to do multiplierless convolution operation and second is parameterized DWT and IDWT computation. Fig. 2 clearly supports that our approach is more memory efficient. When frame length is between 4 to 8 , though memory requirement grows exponentially in our approach, still it saves $50 \%$ of memory than the conventional approach. When frame length is more than 8 , the memory requirement becomes almost linear and saves $90 \%$ of memory than conventional approach. It makes our architecture more effective for longer frames. The proposed architecture requires $2^{7}$ words on-chip memory. This is much less compared to the conventional DA based methods [25] which requires $2^{17}$ words memory to compute 16 point DWT and IDWT keeping the number of filter tap fixed. From architectural point of view, our design is expected to consume 


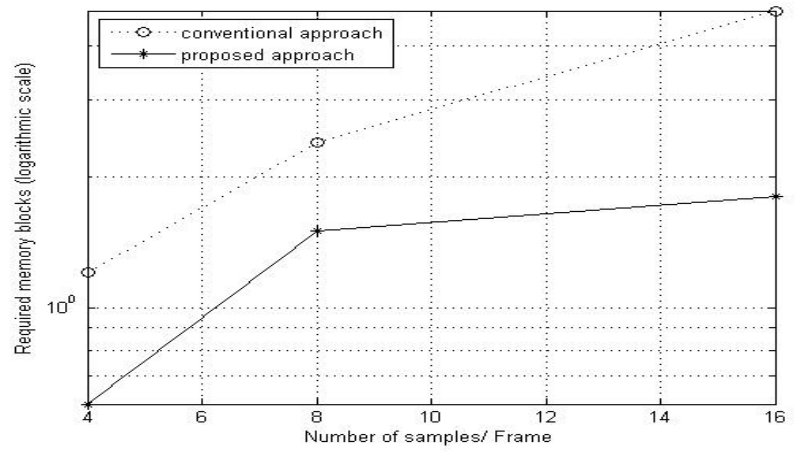

Fig. 2. Required Memory for Conventional DA based approach and Proposed Modified DA based approach [25] (plotted in Logarithmic scale) vs. Number of Samples/ Frame

less power due to significant reduction in memory size and multiplierless implementation.

The architecture described above has been coded in VHDL, synthesized using Synopsys Design Compiler with $0.12 \mu \mathrm{m}$ standard technology for a target frequency of $1 \mathrm{MHz}$ and power has been obtained using Synopsys Prime Time on the synthesized netlist. Total synthesized cell area of the design is $14.2 \mathrm{~mm}^{2}$ and power consumption is $101.5 \mu \mathrm{W}$ at $1.2 \mathrm{~V} @$ $1 \mathrm{MHz}$ frequency.

\section{Conclusion and Future Research Challenges}

We overviewed the recent research efforts in pervasive healthcare system. Significant progress has been made in terms of architecture, sensors, communication and service. We have developed FECG extraction system architecture suitable for pervasive healthcare application, which we believe, makes useful contribution to the Sensor Layer of our proposed threelayered architecture.

There are many outstanding issues that need to be addressed and solutions need to be identified if pervasive healthcare becomes a reality. We believe, the following challenges require innovative research.

Better understanding of the linkage between signal processing algorithms and their impact on architecture implementation is required. Direct application of traditional algorithms will not be viable in architecture level for pervasive healthcare where power and area are the main constraints. So, algorithms have to be designed considering the overall system performance and corresponding architectural implementation issues which may lead to ultra low power circuit design.

\section{REFERENCES}

[1] Y. Liu and F. Li, "PCA: A Reference Architecture for Pervasive Computing", $1^{\text {st }}$ International Symposium on Pervasive Computing and Applications, pp. 99-103, 2006.

[2] I. Korhonen, J. Parkka and M. Gils, "Health Monitoring in the Home of the Future", IEEE Engineering in Medicine and Biology Magazine, vol. 22, no. 3, pp. 66-73, May-June, 2003.

[3] T. L. Hayes, M. Pavel, N. Larimer, I. A. Tsay and J. Nutt, "Distributed Healthcare: Simultaneous Assessment of Multiple Individuals", IEEE Computer Society, Pervasive Computing, pp. 36-43, January- March, 2007.
[4] F. Wang, L. S. Docherty, K. J. Turner, M. Kolberg and E. H. Magill, "Services and Policies for Care At Home", IEEE Pervasive Health Conference and Workshops, pp. 1-10, Nov. 29- Dec. 1, 2006.

[5] N. Doulamis, "An Adaptable Emotionally Rich Pervasive Computing System", European Signal Processing Conference (EUSIPCO), September 4-8, 2006, Italy.

[6] R. Chakravorty, "A Programmable Service Architecture for Mobile Medical Care", $4^{\text {th }}$ Annual IEEE International Conference on pervasive Computing and Communications Workshops (PERCOMW), March, 2006.

[7] Y. V. Reddy, "Pervasive Computing: Implications, Opportunities and Challenges for the Society", $1^{\text {st }}$ International Symposium on Pervasive Computing and Applications, pp. 5-5, 2006.

[8] E. Jovanov, A. Lords, D. Raskovic, P. Cox, R. Adhami and F. Andrasik, "Stress Monitoring Using a Distributed Wireless Intelligent Sensor System", IEEE Engineering in Medicine and Biology Magazine, vol. 22, no. 3, pp. 49-55, May-June, 2003.

[9] K. J. Liszka, M. A. Mackin, M. J. Lichter, D. W. York, D. Pillai and D. S. Rosenbaum, "Keeping a Beat on the Heart", IEEE Pervasive Computing, pp. 42-49, October-December, 2004.

[10] U. Varshney, "Managing Comprehensive Wireless Patient Monitoring", IEEE Pervasive Health Conference and Workshops, pp. 1-4, Nov. 29, 2006.

[11] U. Varshney, "Pervasive Healthcare and Wireless Health Monitoring", Journal of Mobile Networks and Applications, Springer Netherlands, vol. 12, Nov. 2-3, June, 2007.

[12] J. A. Muras, V. Cahill and E. K. Stokes, "A Taxonomy of Pervasive Healthcare Systems", IEEE Pervasive Health Conference and Workshops, pp. 1-10, Nov. 29- dec. 1, 2006.

[13] M. Weiser, "The Computer for the $21^{\text {st }}$ Century", IEEE Pervasive Computing, vol. 1, issue. 1, pp. 19-25, Jan-March, 2002.

[14] S. Tilak, N. B. Abu-Ghazaleh and W. Heinzelman, "A Taxonomy of Wireless Micro-Sensor Network Models", SIGMOBILE Mob. Comput. Commun. Rev., vol. 6, no. 2, pp. 28-36, 2002.

[15] S. Cheekiralla and D. W. Engels, "A Functional Taxonomy of Wireless Sensor Network Devices", $2^{\text {nd }}$ IEEE/ CreateNet Workshop on Broadband Advanced Sensor Networks (BaseNetS), October, 2005.

[16] I. F. Akyildiz, W. Su, Y. Sankarasubramaniam and E. Cayirci, "A Survey on Sensor Networks", IEEE Communications Magazine, no. 2, pp. 102114, August 2002.

[17] G. J. Pottie and W. J. Kaiser, "Wireless Integrated Network Sensors", Communications of the ACM, vol. 43, no. 5, pp. 51-58, May, 2000.

[18] D. Estrin, L. Girod, G. Pottie and M. Srivastava, "Instrumenting the World with Wireless Sensor Networks", IEEE International Conference on Acoustics, Speech and Signal Processing (ICASSP), vol. 4, pp. 20332036, 2001.

[19] I. F. Akyildiz, W. Su, Y. Sankarasubramaniam and E. Cayirci, "Wireless Sensor Networks: A Survey", Computer Networks, vol. 38, no. 4, pp. 393-422, 2002.

[20] D. Estrin, D. Culler, K. Pister and G. Sukhatme, "Connecting the Physical World with Pervasive Networks", IEEE Pervasive Computing, vol. 1, no. 1, pp. 59-69, 2002.

[21] P. Celka, R. Vetter, P. Renevey, C. Verjus, V. Neuman, J. Luprano, J. Decotignie and C. Piguet, "Wearable Biosensing: Signal processing and Communication", Journal of Telecommunications and Information Technology, vol. 4, pp. 90-104, 2005.

[22] R. G. Maunder, A. S. Weddell. G. V. Merrett, B. M. Al-Hashimi and L. Hanzo, "Iterative Decoding for Redistributing Energy Consumption in Wireless Sensor Networks", International Conference on Computer Communications and Networks, August 3-7, 2008, St. Thomas, Virgin Islands, USA

[23] H. Hassanpour and A. Parsaei, "Fetal ECG Extraction Using Wavelet Transform", IEEE Computer Society CIMCA and IAWTIC, 2006.

[24] A. Khamene and S. Negahdaripour, "A New Method for the Extraction of Fetal ECG from the Composite Abdominal Signal", IEEE Trans. on Biomedical Engineering, vol. 47, no. 4, pp. 507-516, April, 2000.

[25] S. Ramprasad, N. R. Shanbhag and I. N. Hajj, "Low-Power Distributed Arithmetic Architectures Using Non-Uniform Memory Partitioning", IEEE International Symposium on Circuits and Systems, vol. 3, pp. 470473, July, 1999. 